\title{
Carbon C 11 Methylaminoisobutyrate
}

National Cancer Institute

\section{Source}

National Cancer Institute. Carbon C 11 Methylaminoisobutyrate. NCI Thesaurus. Code C118360.

A synthetic neutral amino acid analog radiolabeled with carbon C 11 with potential use for metabolic tumor imaging during positron emission tomography (PET). Upon administration, carbon C 11 methylaminoisobutyrate $([(11) \mathrm{C}] \mathrm{MeAIB})$ is taken up by and accumulates in cancer cells through the A-type (alanine-preferring) amino acid transport system. Upon imaging of the radioisotope, the distribution patterns of MeAIB and its uptake by cancer cells can be further elucidated. This may establish its usefulness as a tumor tracer in the diagnosis of certain cancers. Amino acid transport system A is highly upregulated in malignant cells and facilitates the uptake of amino acids needed for tumor cell proliferation. 